\title{
Multilayer tube impedance and external radiation
}

\author{
M. Ivanyan, E. Laziev, V. Tsakanov, and A. Vardanyan \\ CANDLE, Yerevan State University, Acharyan 31, 0040, Yerevan, Armenia \\ S. Heifets \\ SLAC, Stanford University, 2575 Sand Hill Road, Menlo Park, California 94025, USA
}

A. Tsakanian

Yerevan Physics Institute, Alikhanian Br. 2, 0036, Yerevan, Armenia, and Institute of Experimental Physics, Hamburg University, Luruper Chaussee 149, 22761, Hamburg, Germany (Received 3 September 2007; revised manuscript received 28 March 2008; published 13 August 2008; corrected 27 August 2008)

The paper describes a method for calculating the longitudinal and transverse impedances of the laminated round pipe with many layers of different materials. The charge is moving along the pipe axis with arbitrary constant velocity. The study is based on the field-matching technique applied for the arbitrary harmonic of the electromagnetic field. The matrix formalism has been developed to describe the field transitions through the subsequent layers that allow coupling the electromagnetic fields inside and outside the pipe. The number of equations to be solved is then reduced to four algebraic equations. The solutions and ultrarelativistic limits for the field harmonics in the inner and outer regions of the pipe are derived.

DOI: 10.1103/PhysRevSTAB.11.084001

PACS numbers: 41.60. $-\mathrm{m}, 41.75 . \mathrm{Ht}, 42.82 . \mathrm{tt}$

\section{INTRODUCTION}

The development of advanced accelerators is accompanied with the improvement and modification of the facility characteristics that require careful modeling and examination of the effects that impact the beam quality [1]. The longitudinal and transverse impedances of the accelerator vacuum chamber caused by finite conductivity of the wall material are important characteristics of the facility performance describing the interaction of the charged particle beam with the chamber walls in the frequency domain [1,2].

In advanced electron, hadron, or heavy ion accelerators [3-9], the technical solutions for high vacuum performance, cures of resistive instabilities [10-12], prevention of the static charge $[13,14]$, and shielding of external radiation [15-19] very often imply the usage of laminated (or multilayer) vacuum chamber configuration. These complications along with the stringent requirements to the beam quality recently drove rigorous study of the longitudinal and transverse resistive impedances of the multilayer vacuum chamber to obtain the exact analytical solution valid for both nonrelativistic and ultrarelativistic beams [1822].

The general approach to solve Maxwell equations for the multilayer tube is a field-matching technique [1,2,1016,18-22]. The classical ultrarelativistic formulas [1,23], usually applied for the ordinary vacuum chamber impedance calculation, were derived for the vacuum chamber of infinite wall thickness and generalized for the case of the finite-thickness metallic [1] or infinite-thickness ceramic wall [2]. For the arbitrary number of layers, the recurrent method based on the numerical consequent solutions of the matching equations is described in [2,24-26]. The transverse dipole impedance for the two-layer tube with infinite external layer thickness is derived in [26]. A number of analytical and numerical studies of multilayer tube impedance are given in [17,22,27-32]. In [20,21] the analytical solutions for longitudinal and transverse impedances of the two-layer tube in the ultrarelativistic case have been derived. However, even the case of the three-layer tube causes difficulties for direct calculations [22,31,32].

The plain disklike charge with variable density over radius and azimuth was taken as a basic model of a driving charge in works $[1,2,16,18-21,23]$. In the ultrarelativistic limit, the results of the model are interpreted as the multipole expansion of the results for a pointlike charge impedance [21,33], but for a nonrelativistic beam the azimuthal harmonics of the fields generated by disklike charge substantially differ from the field harmonics of a pointlike charge.

In this paper we describe a method for calculating the longitudinal and transverse impedances of the point charge moving with arbitrary constant velocity along the axis of the laminated round pipe with many layers of different materials. The charge fields inside the pipe are presented by the superposition of the field azimuthal harmonics. To evaluate the effect of the laminated vacuum chamber, the field transformation matrix concept is introduced to couple the tangential components of the electromagnetic fields between the borders of subsequent layers. The transformation matrix defined by geometrical and material characteristics of the layer then describes each single layer. The transformation matrix formalism allows direct matching of the electromagnetic fields inside and outside the pipe thus 
reducing the number of equations to four algebraic equations for arbitrary number of layers. The solutions and ultrarelativistic limits for the field harmonics in the inner and outer regions of the pipe are derived. The numerical examples for the PETRA III vacuum chamber [34] and three-layer tube are presented.

\section{FIELD PRESENTATION AND SOLUTION METHOD}

Consider a circular-cylindrical many-layer tube of inner $a_{1}$ and outer $a_{N+1}$ radii (Fig. 1). The layers can be either dielectrics or metals and are characterized by the dielectric permittivity $\varepsilon_{i}$ and magnetic permeability $\mu_{i} \quad(i=$ $1,2,3, \ldots, N)$. The pointlike charge $q$ with the offset $r_{q}$ is moving in parallel to the $z$ axis with the constant velocity $v$. We will use cylindrical coordinates $\vec{r}=(r, z, \theta)$ and assume that in inner $\left(0 \leq r<a_{1}\right)$ and outer $\left(r>a_{N+1}\right)$ regions of the pipe are vacuum.

The problem is solved by the field-matching technique that implies the continuity of the tangential components of electric and magnetic fields at the borders of the layers. As it follows from the geometry of the vacuum chamber (Fig. 1), for the $N$ layer tube there are $N+2$ regions (including inner and outer regions of the tube) and $N+1$ borders. Four boundary equations should be composed for each border that match the $E_{\theta}, E_{z}, B_{\theta}$, and $B_{z}$ components of electromagnetic field, thus the complete number of matching equations is $4(N+1)$ with $4(N+1)$ unknown coefficients.

In general, due to current axial asymmetry, the fields radiated in the tube have all six components $E_{z}, B_{z}, E_{\theta}, B_{\theta}$, $E_{r}, B_{r}$. As usual, for cylindrical geometry we will use the multipole presentation of the charge distribution and electromagnetic fields and will consider only the synchronous components of the fields. The frequency domain multipole components of the electromagnetic fields, synchronously moving with the charge, can be then represented as [1,2]

$$
\begin{aligned}
& \left\{E_{r}, E_{z}, B_{\theta}\right\}_{m}(\omega, \vec{r})=\left\{E_{r}, E_{z}, B_{\theta}\right\}_{m}(\omega, r) \cos m \theta \exp (j k z) \\
& \left\{B_{r}, B_{z}, E_{\theta}\right\}_{m}(\omega, \vec{r})=\left\{B_{r}, B_{z}, E_{\theta}\right\}_{m}(\omega, r) \sin m \theta \exp (j k z) .
\end{aligned}
$$

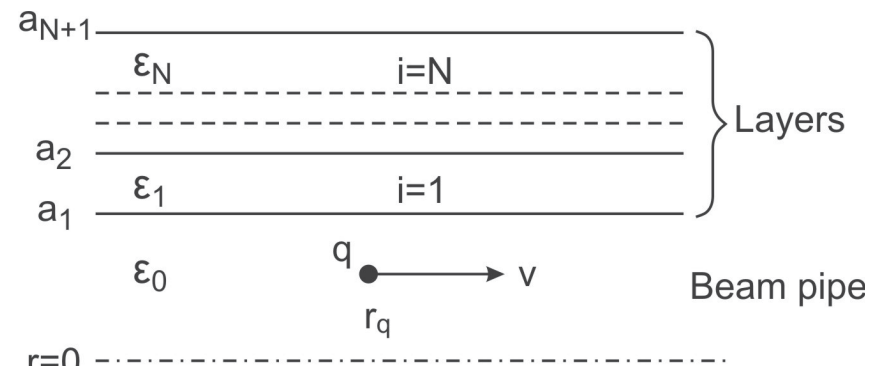

FIG. 1. Geometry of the laminated vacuum chamber. The pointlike charge is moving with arbitrary constant velocity along the tube axis. where $\omega$ is the frequency and $k=\omega / v$ is the wave number.

The $m$-pole component of charge distribution is given by

$$
\rho_{m}(\omega, \vec{r})=\frac{q h_{m}}{\pi r_{q} v} \delta\left(r-r_{q}\right) \cos m \theta \exp (j k z),
$$

with $h_{0}=1 / 2$ and $h_{m}=1$ for $m>0$. We will omit the factors $\exp (j k z), \cos m \theta$, and $\sin m \theta$ from further considerations and consider the radial dependence of the $m$ azimuthal pole fields in the frequency domain.

The geometry of the problem is composed of three regions (Fig. 1), where the fields are expressed in different ways: the charge existing region $\left(0 \leq r \leq a_{1}\right)$, the laminated wall of the tube $\left(a_{1} \leq r \leq a_{N+1}\right)$, and the outer vacuum region $\left(r \geq a_{N+1}\right)$. The matching conditions on the borders of the layers $r=a_{i+1}(i=0,1,2 \ldots, N)$, i.e., the continuity of tangential components of electric and magnetic fields, are read as

$$
\begin{aligned}
E_{\theta m}^{(i)}\left(a_{i+1}\right) & =E_{\theta m}^{(i+1)}\left(a_{i+1}\right), \\
E_{z m}^{(i)}\left(a_{i+1}\right) & =E_{z m}^{(i+1)}\left(a_{i+1}\right), \\
B_{\theta m}^{(i)}\left(a_{i+1}\right) / \mu_{i}^{\prime} & =B_{\theta m}^{(i+1)}\left(a_{i+1}\right) / \mu_{i+1}^{\prime}, \\
B_{z m}^{(i)}\left(a_{i+1}\right) / \mu_{i}^{\prime} & =B_{z m}^{(i+1)}\left(a_{i+1}\right) / \mu_{i+1}^{\prime}
\end{aligned}
$$

with $\mu_{i}^{\prime}=\mu_{i} / \mu_{0}$ the relative magnetic permeability of the $i$ th region.

We will use the complete solution for the pipe with perfectly conducting walls as a partial solution in the inner part of the pipe $[1,35]$. The radial dependence of the partial solution for the $m$-pole mode is given by

$$
E_{z m}^{(p)}(r)=\grave{A}_{0} \tau \lambda\left[I_{m}(\lambda r) C+P\left(r_{q}, r\right)\right],
$$

with

$$
\begin{aligned}
C & =-I_{m}\left(\lambda r_{q}\right) K_{m}\left(\lambda a_{1}\right) / I_{m}\left(\lambda a_{1}\right), \\
P\left(r_{q}, r\right) & = \begin{cases}K_{m}\left(\lambda r_{q}\right) I_{m}(\lambda r), & r \leq r_{q} \\
I_{m}\left(\lambda r_{q}\right) K_{m}(\lambda r), & r \geq r_{q},\end{cases}
\end{aligned}
$$

and $Z_{0}=120 \pi \Omega, \beta=v / c, \grave{A}_{0}=-j \frac{q Z_{0} h_{m}}{\pi \beta}, \tau=\gamma^{-1}$, $\lambda=k / \gamma$ with $\gamma$ the particle Lorenz factor. Other components of electromagnetic field are derived as

$$
\begin{aligned}
E_{r m}^{(p)}(r) & =-j A_{0} \lambda\left[I_{m}^{\prime}(\lambda r) C+\frac{\partial}{\partial r} P\left(r_{q}, r\right)\right], \\
E_{\theta m}^{(p)}(r) & =j A_{0} \frac{m}{r}\left[I_{m}(\lambda r) C+P\left(r_{q}, r\right)\right], \\
B_{r m}^{(p)}(r) & =-\beta^{2} E_{\theta m}^{(p)}(r) / v, \\
B_{\theta m}^{(p)}(r) & =\beta^{2} E_{r m}^{(p)}(r) / v, \\
B_{z m}^{(p)} & =0 .
\end{aligned}
$$

Note that the radial electric and azimuthal magnetic components of the solutions (5) have discontinuity at the offset 
$r=r_{q}$ due to current and charge densities formed by the pointlike charge.

The solution of the homogeneous wave equation in the inner region of the pipe, which includes the axis $r=0$, is only the modified Bessel functions of the first kind since those of the second kind diverge for argument zero. We will be interested about the tangential (longitudinal and azimuthal) components of the electromagnetic fields and introduce the tangential field vector $\hat{T}_{m}\left(E_{\theta m}\right.$, $\left.E_{z m}, j v B_{\theta m} / \mu^{\prime}, j v B_{z m} / \mu^{\prime}\right)$. The complete solution in the inner part of the tube in vector form is then presented as

$$
\hat{T}_{m}^{\text {in }}(r)=D_{1} \hat{V}_{1}(r)+D_{2} \hat{V}_{2}(r)+\hat{R}^{(p)}(r), \quad r \leq a_{1},
$$

where

$$
\hat{V}_{1}=\left(\begin{array}{c}
-I_{m}^{\prime}(\lambda r) \\
0 \\
\frac{j m}{\lambda r} I_{m}(\lambda r) \\
-\tau I_{m}(\lambda r)
\end{array}\right), \quad \hat{V}_{2}=\left(\begin{array}{c}
\frac{j m}{\lambda r} I_{m}(\lambda r) \\
\tau I_{m}(\lambda r) \\
\beta^{2} I_{m}^{\prime}(\lambda r) \\
0
\end{array}\right),
$$

with $D_{1}, D_{2}$ uncertain coefficients and the components of the vector $\hat{R}^{(p)}\left(E_{\theta m}^{(p)}, E_{z m}^{(p)}, j v B_{\theta m}^{(p)} / \mu^{\prime}, j v B_{z m}^{(p)} / \mu^{\prime}\right)$ are given by the partial solution (4) and (5). The general solution in (6) describes electromagnetic fields induced in the tube due to the wall's finite conductivity.

The fields in the wall layers $a_{i} \leq r \leq a_{i+1} \quad(i=$ $1,2 \ldots, N$ ) include both kinds of modified Bessel function. Introducing the vector of unknown coefficients $\hat{A}^{(i)}\left(A_{1}^{(i)}, A_{2}^{(i)}, A_{3}^{(i)}, A_{4}^{(i)}\right)$, the solution for tangential components in the $i$ th layer can be presented in matrix form as

$$
\hat{T}^{(i)}\left(\chi_{i} r\right)=\hat{W}\left(\chi_{i} r\right) \hat{A}^{(i)}
$$

where

$$
\hat{W}\left(\chi_{i} r\right)=\left\{\begin{array}{cccc}
-K_{m}^{\prime}\left(\chi_{i} r\right) & -I_{m}^{\prime}\left(\chi_{i} r\right) & \frac{j m}{\chi_{i} r} K_{m}\left(\chi_{i} r\right) & \frac{j m}{\chi_{i} r} I_{m}\left(\chi_{i} r\right) \\
0 & 0 & \frac{\chi_{i}}{k} K_{m}\left(\chi_{i} r\right) & \frac{\chi_{i}}{k} I_{m}\left(\chi_{i} r\right) \\
\frac{j m}{\mu_{i}^{\prime} \chi_{i} r} K_{m}\left(\chi_{i} r\right) & \frac{j m}{\mu_{i}^{\prime} \chi_{i} r} I_{m}\left(\chi_{i} r\right) & \varepsilon_{i}^{\prime} \beta^{2} K_{m}^{\prime}\left(\chi_{i} r\right) & \varepsilon_{i}^{\prime} \beta^{2} I_{m}^{\prime}\left(\chi_{i} r\right) \\
-\frac{\chi_{i}}{k \mu_{i}^{\prime}} K_{m}\left(\chi_{i} r\right) & -\frac{\chi_{i}}{k \mu_{i}^{\prime}} I_{m}\left(\chi_{i} r\right) & 0 & 0
\end{array}\right\} .
$$

Here $\varepsilon_{i}^{\prime}=\varepsilon_{i} / \varepsilon_{0}, \mu_{i}^{\prime}=\mu_{i} / \mu_{0}$ are the relative permittivity and the permeability with respect to vacuum constants $\varepsilon_{0}$, $\mu_{0}$, and $\chi_{i}=\sqrt{k^{2}-\mu_{i} \varepsilon_{i} \omega^{2}}\left[\operatorname{Re}\left(\chi_{i}\right)>0\right]$ are the radial propagation constants in the $i$ th layer.

In the outer region of the pipe $\left(r \geq a_{N+1}\right)$ that extends to infinity, only modified Bessel functions of the second kind are admissible. The solution for tangential components is read as

$$
\hat{T}_{m}^{\text {out }}(r)=D_{3} \hat{V}_{3}(r)+D_{4} \hat{V}_{4}(r), \quad r \geq a_{N+1},
$$

where

$$
\hat{V}_{3}=\left(\begin{array}{c}
-K_{m}^{\prime}(\lambda r) \\
0 \\
\frac{j m}{\lambda r} K_{m}(\lambda r) \\
-\tau K_{m}(\lambda r)
\end{array}\right), \quad \hat{V}_{4}=\left(\begin{array}{c}
\frac{j m}{\lambda r} K_{m}(\lambda r) \\
\tau K_{m}(\lambda r) \\
\beta^{2} K_{m}^{\prime}(\lambda r) \\
0
\end{array}\right) .
$$

The inner $\left(D_{1}, D_{2}\right)$ and external $\left(D_{3}, D_{4}\right)$ field coefficients are determined by matching the tangential components of the fields on the borders of the layers according to (3).

\section{FIELDS TRANSFORMATION MATRIX}

In this chapter we introduce the fields transformation matrix $\hat{Q}^{(i)}$ to connect the tangential components of electromagnetic fields at the bottom surfaces of two subsequent layers $i$ and $i+1$ to exclude the uncertain coefficients in
Eq. (8) from further consideration. Using the field presentation (8), the tangential components of the fields at the bottom $\left(r=a_{i}\right)$ and top $\left(r=a_{i+1}\right)$ surfaces of the $i$ th layer are related as

$$
\hat{T}_{m}^{(i)}\left(\chi_{i} a_{i}\right)=\hat{Q}^{(i)} \hat{T}_{m}^{(i)}\left(\chi_{i} a_{i+1}\right),
$$

where $\hat{Q}^{(i)}=\hat{W}\left(\chi_{i} a_{i}\right) \cdot \hat{W}^{-1}\left(\chi_{i} a_{i+1}\right)$ is the field transformation matrix through the $i$ th layer given by

$$
\hat{Q}^{(i)}=\left(\begin{array}{cccc}
q_{11} & q_{12} & -\alpha_{0} q_{31} & \alpha_{0} q_{32} \\
0 & q_{22} & \alpha_{0} q_{41} & -\alpha_{0} q_{42} \\
q_{31} & q_{32} & q_{11} & -q_{12} \\
q_{41} & q_{42} & 0 & q_{22}
\end{array}\right)
$$

with $\alpha_{0}=\frac{\mu_{i}^{\prime}}{\varepsilon_{i}^{\prime} \beta^{2}}$. The seven independent elements of the field transformation matrix $\hat{Q}^{(i)}$ are determined by corresponding layer geometrical and electromagnetic parameters and are given as 


$$
\begin{aligned}
& q_{11}=-a_{i+1} \chi_{i} U_{3}^{(i)}, \\
& q_{22}=a_{i+1} \chi_{i} U_{2}^{(i)}, \\
& q_{12}=-\frac{j k m}{a_{i} \chi_{i}^{2}}\left(\frac{a_{i}}{a_{i+1}} q_{11}-q_{22}\right), \\
& q_{42}=\frac{j}{\mu_{i}^{\prime}} m U_{1}^{(i)}, \\
& q_{32}=k\left(\beta^{2} \varepsilon_{i}^{\prime} a_{i+1} U_{4}^{(i)}+\frac{m^{2} U_{1}^{(i)}}{\mu_{i}^{\prime} a_{i} \chi_{i}^{2}}\right), \\
& q_{41}=-\frac{a_{i+1} \chi_{i}^{2}}{k \mu_{i}^{\prime}} U_{1}^{(i)}, \\
& q_{31}=\frac{a_{i+1}}{a_{i}} q_{42},
\end{aligned}
$$

where $U_{k}^{(i)}(k=1,2,3,4)$ are the combinations of modified Bessel functions of both kinds:

$$
\begin{aligned}
U_{1}^{(i)} & =K_{m}\left(\chi_{i} a_{i}\right) I_{m}\left(\chi_{i} a_{i+1}\right)-I_{m}\left(\chi_{i} a_{i}\right) K_{m}\left(\chi_{i} a_{i+1}\right) \\
& \approx \sinh \left(\chi_{i} d_{i}\right) / \chi_{i} \sqrt{a_{i+1} a_{i}}, \\
U_{2}^{(i)} & =K_{m}\left(\chi_{i} a_{i}\right) I_{m}^{\prime}\left(\chi_{i} a_{i+1}\right)-I_{m}\left(\chi_{i} a_{i}\right) K_{m}^{\prime}\left(\chi_{i} a_{i+1}\right) \\
& \approx \cosh \left(\chi_{i} d_{i}\right) / \chi_{i} \sqrt{a_{i+1} a_{i}}, \\
U_{3}^{(i)} & =K_{m}^{\prime}\left(\chi_{i} a_{i}\right) I_{m}\left(\chi_{i} a_{i+1}\right)-I_{m}^{\prime}\left(\chi_{i} a_{i}\right) K_{m}\left(\chi_{i} a_{i+1}\right) \\
& \approx-\cosh \left(\chi_{i} d_{i}\right) / \chi_{i} \sqrt{a_{i+1} a_{i}}, \\
U_{4}^{(i)} & =K_{m}^{\prime}\left(\chi_{i} a_{i}\right) I_{m}^{\prime}\left(\chi_{i} a_{i+1}\right)-I_{m}^{\prime}\left(\chi_{i} a_{i}\right) K_{m}^{\prime}\left(\chi_{i} a_{i+1}\right) \\
& \approx-\sinh \left(\chi_{i} d_{i}\right) / \chi_{i} \sqrt{a_{i+1}} a_{i} .
\end{aligned}
$$

In the frequency range where the skin depth of a layer is much smaller than the inner radius of the tube, i.e., for all the practically important cases, the functions $U_{k}^{(i)}$ can be approximated by hyperbolic functions as is given in (15) to construct the asymptotic expressions of impedance [2022,26]. In (15) $d_{i}=a_{i+1}-a_{i}$ is the $i$ th layer thickness.

All elements of the matrix $\hat{Q}^{(i)}(13)$ are dimensionless and the determinant of the matrix is equal to $a_{i+1}^{2} / a_{i}^{2}$. As is seen from (14) for the monopole term $m=0$, the following elements of field transformation matrix $q_{12}=q_{13}=$ $q_{21}=q_{24}=q_{31}=q_{34}=q_{42}=q_{43} \equiv 0$ are vanishing. For the zero thickness layer the field transformation matrix is transformed to unit matrix.

Taking into account the matching condition at the border of two subsequent layers $\hat{T}_{m}^{(i)}\left(\chi_{i} a_{i+1}\right)=\hat{T}_{m}^{(i+1)}\left(\chi_{i+1} a_{i+1}\right)$, the tangential components of electromagnetic fields at the inner $\left(r=a_{1}\right)$ and outer $\left(r=a_{N+1}\right)$ surfaces of the pipe are related as

$$
\begin{aligned}
\hat{T}_{m}^{\text {in }}\left(r=a_{1}\right) & =\hat{T}_{m}^{(1)}\left(\chi_{1} a_{1}\right) \\
& =\hat{Q}^{(1)} \cdot \hat{Q}^{(2)} \cdots \hat{Q}^{(N)} \hat{T}^{(N)}\left(\chi_{N} a_{N+1}\right) \\
& =\hat{Q} \hat{T}_{m}^{\text {out }}\left(r=a_{N+1}\right),
\end{aligned}
$$

where

$$
\hat{Q}=\hat{Q}^{(1)} \hat{Q}^{(2)} \cdots \hat{Q}^{(N)}
$$

is the total field transformation matrix through the pipe laminated wall. The matching equations in matrix form now are read as

$$
\hat{T}_{m}^{\mathrm{in}}\left(r=a_{1}\right)=\hat{Q} \hat{T}_{m}^{\mathrm{out}}\left(r=a_{N+1}\right)
$$

that contain only four unknown coefficients $\left(D_{1}, D_{2}, D_{3}, D_{4}\right)$ given by the solutions in inner (6) and outer (10) regions of the pipe.

\section{FIELD COEFFICIENTS DETERMINATION}

The tangential components of the full field in the inner and outer surfaces of the pipe are given by the solutions (6) and (10), respectively, and can be presented in matrix form. In particular, for the tangential components of the field on the inner surface of the pipe $\left(r=a_{1}\right)$, one can write

$$
\hat{T}_{m}^{\text {in }}=\hat{V}_{1} D_{1}+\hat{V}_{2} D_{2}+\hat{R}^{(p)}, \quad r=a_{1},
$$

where

$$
\hat{R}^{(p)}\left(a_{1}\right)=\left(\begin{array}{c}
0 \\
0 \\
\grave{A}_{0} \frac{I_{m}\left(\lambda r_{q}\right)}{a_{1}} \frac{I_{m}\left(\lambda a_{1}\right)}{0} \\
0
\end{array}\right) .
$$

Following (10) the external field's tangential components on the outer surface of the pipe in matrix form can be presented as follows:

$$
\hat{T}_{m}^{\text {out }}=D_{3} \hat{V}_{3}+D_{4} \hat{V}_{4}, \quad r=a_{N+1} .
$$

Using the above presentations for the tangential components of the field in the inner and outer surfaces of the pipe, Eq. (19) is modified to

$$
\hat{V}_{1} D_{1}+\hat{V}_{2} D_{2}+\hat{R}^{(p)}=\hat{Q}\left(\hat{V}_{3} D_{3}+\hat{V}_{4} D_{4}\right) .
$$

Introducing the column of unknown coefficients $\hat{D}\left(D_{1}, D_{2}, D_{3}, D_{4}\right)$, Eq. (21) in matrix form is read as

$$
\hat{S} \cdot \hat{D}=-\hat{R}^{(p)},
$$

where $\hat{S}$ is $4 \times 4$ matrix composed as

$$
\hat{S}=\left\{\hat{V}_{1}, \hat{V}_{2},-\hat{Q} \cdot \hat{V}_{3},-\hat{Q} \cdot \hat{V}_{4}\right\} .
$$

The solution for four unknown coefficients in matrix form is then given by

$$
\hat{D}=-\hat{S}^{-1} \hat{R}^{(p)} .
$$

Substituting coefficients determined by (24) into field component expressions (6) and (10), we obtain the radiated fields in the inner and outer regions of the pipe for arbitrary harmonic number. The field components inside and outside of the pipe are then expressed as 


$$
\begin{aligned}
E_{z m}^{\mathrm{in}}= & \tau I_{m}(\lambda r) D_{2}+E_{z}^{(p)} \\
v B_{z m}^{\mathrm{in}}= & j \tau I_{m}(\lambda r) D_{1} \\
E_{m \theta, r}^{\text {in }}= & -\left\{\left(D_{1}+j D_{2}\right) I_{m+1}(\lambda r)\right. \\
& \left. \pm\left(D_{1}-j D_{2}\right) I_{m-1}(\lambda r)\right\} / 2+E_{\theta, r}^{(p)} \\
v B_{m \theta, r}^{i n}= & -\left\{ \pm\left(D_{1}+j \beta^{2} D_{2}\right) I_{m+1}(\lambda r)\right. \\
& \left.-\left(D_{1}-j \beta^{2} D_{2}\right) I_{m-1}(\lambda r)\right\} / 2+v B_{\theta, r}^{(p)},
\end{aligned}
$$

and

$$
\begin{aligned}
E_{m z}^{\text {out }}(\lambda r)= & \tau D_{4} K_{m}(\lambda r), \\
j v B_{m z}^{\text {out }}(\lambda r)= & -\tau D_{3} K_{m}(\lambda r) \\
E_{m \theta, r}^{\text {out }}= & \left\{\left(D_{3}+j D_{4}\right) K_{m+1}(\lambda r)\right. \\
& \left. \pm\left(D_{3}-j D_{4}\right) K_{m-1}(\lambda r)\right\} / 2 \\
v B_{m \theta, r}^{\text {out }}= & \left\{ \pm\left(D_{3}+j \beta^{2} D_{4}\right) K_{m+1}(\lambda r)\right. \\
& \left.-\left(D_{3}-j \beta^{2} D_{4}\right) K_{m-1}(\lambda r)\right\} / 2 .
\end{aligned}
$$

In (25) and (26) the plus stands for the $\theta$ component, and minus for the $r$ component of the field.

In particular, for monopole mode $(m=0)$, the field coefficients are derived as

$$
\begin{aligned}
& D_{1}=0 \text {, } \\
& D_{3}=0 \text {, } \\
& D_{2}=-\frac{j q Z_{0}}{2 \pi} \frac{I_{0}\left(\lambda r_{q}\right)}{a_{1} I_{0}\left(\mathrm{~s}_{1}\right)} \cdot \frac{\beta}{I_{0}^{\prime}\left(\mathrm{s}_{1}\right) \beta^{2}-\tau I_{0}\left(\mathrm{~s}_{1}\right) \alpha}, \\
& D_{4}=-\frac{j q Z_{0}}{2 \pi} \frac{I_{0}\left(\lambda r_{q}\right)}{a_{1} I_{0}\left(\mathrm{~s}_{1}\right)} \cdot \frac{\tau \beta}{Q_{23} K_{0}^{\prime}\left(\mathrm{s}_{2}\right) \beta^{2}+Q_{22} K_{0}\left(\mathrm{~s}_{2}\right) \tau} \\
& \cdot \frac{1}{I^{\prime}\left(\mathrm{s}_{2}\right) \beta^{2}-\tau I_{0}\left(\mathrm{~s}_{1}\right) \alpha} \text {, } \\
& \text { with } \mathrm{s}_{1}=\lambda a_{1}, \mathrm{~s}_{2}=\lambda a_{N+1} \text {, and } \\
& \alpha=\frac{Q_{33} \beta^{2} K_{0}^{\prime}\left(\mathrm{s}_{2}\right)+Q_{32} \tau K_{0}\left(\mathrm{~s}_{2}\right)}{Q_{23} \beta^{2} K_{0}^{\prime}\left(\mathrm{s}_{2}\right)+Q_{22} \tau K_{0}\left(\mathrm{~s}_{2}\right)} .
\end{aligned}
$$

The field coefficients for high multipole modes $(m>0)$ expressed via the elements $Q_{i j}$ of field transformation matrix $\hat{Q}$ are given in Appendix A.

\section{ULTRARELATIVISTIC CASE}

The ultrarelativistic limit of the induced fields is derived by $\tau=\gamma^{-1} \rightarrow 0$. Ultrarelativistic expressions of the partial solutions (4) and (5) are given by

$$
\begin{aligned}
& E_{z m}^{(p)}=B_{z m}^{(p)}=0, \quad(m \geq 0) \\
& E_{\theta 0}^{(p)}=B_{r 0}^{(p)}=0 \text {, } \\
& E_{r 0}^{(p)}=c B_{\theta 0}^{(p)}=\left\{\begin{array}{ll}
q Z_{0} / 2 \pi r, & r \geq r_{q} \\
0 & r<r_{q}
\end{array}, \quad(m=0)\right. \\
& E_{\theta m}^{(p)}=-c B_{r m}^{(p)}=\frac{q Z_{0}}{2 \pi}\left[-\frac{r^{m-1} r_{q}^{m}}{a_{1}^{2 m}}+\left\{\begin{array}{ll}
r_{q}^{m} / r^{m+1}, & r \geq r_{q} \\
r^{m-1} / r_{q}^{m}, & r \leq r_{q}
\end{array}\right], \quad(m>0)\right. \\
& E_{r m}^{(p)}=c B_{\theta m}^{(p)}=\frac{q Z_{0}}{2 \pi}\left[\frac{r^{m-1} r_{q}^{m}}{a_{1}^{2 m}}+\left\{\begin{array}{ll}
r_{q}^{m} / r^{m+1}, & r \geq r_{q} \\
-r^{m-1} / r_{q}^{m} & r<r_{q}
\end{array}\right], \quad(m>0) .\right.
\end{aligned}
$$

The monopole term $(m=0)$ of radiated fields in the ultrarelativistic limit is explicitly expressed through the transformation matrix elements both in inner and outer regions of the pipe.

Inner region:

$$
\begin{aligned}
& E_{z}^{\text {in }}=-j \frac{q Z_{0}}{\pi k a_{1}^{2}}\left(1-\frac{2}{k a_{1}} \frac{Q_{33}}{Q_{23}}\right)^{-1}, \quad B_{z}^{\text {in }}=B_{r}^{\text {in }}=E_{\theta}^{\text {in }} \equiv 0, \\
& E_{r}^{\text {in }}=c B_{\theta}^{\text {in }}=-\frac{q Z_{0} r}{2 \pi a_{1}^{2}}\left(1-\frac{2}{k a_{1}} \frac{Q_{33}}{Q_{23}}\right)^{-1}+ \begin{cases}0, & r<r_{q} \\
j \frac{q Z_{0}}{\pi r}, & r>r_{q}\end{cases}
\end{aligned}
$$

Outer region:

$$
E_{r}^{\text {out }}=v B_{\theta}^{\text {out }}=-\frac{q Z_{0}}{2 \pi a_{1}}\left(Q_{33}-\frac{k a_{1}}{2} Q_{23}\right)^{-1} \frac{a_{N+1}}{r} \quad E_{z}^{\text {out }}=E_{\theta}^{\text {out }}=B_{z}^{\text {out }}=B_{r}^{\text {out }}=0 .
$$

The asymptotic presentation of dipole mode $(m=1)$ for the large particle Lorenz factor contains logarithmical terms slowly varying with $\gamma$. Therefore, the correct ultrarelativistic approximation of the dipole mode for the large but finite particle Lorenz factor is the asymptotic presentation of the fields 


$$
\begin{aligned}
E_{z}^{\text {in }} & =-c B_{z}^{\text {in }}=-j \frac{q Z_{0} k}{\pi}\left(\frac{k^{2} a_{1}^{2}}{2}-1+k a_{1} V^{+} / V^{-}\right)^{-1} \frac{r_{q}}{a_{1}} \frac{r}{a_{1}} \\
E_{z}^{\text {out }} & =c B_{z}^{\text {in }}=j \frac{q Z_{0} k}{\pi} \frac{k a_{N+1}\left(Q_{23}-Q_{41}-j Q_{21}-j Q_{43}\right)}{2 k a_{1} V^{+}+\left(k^{2} a_{1}^{2}-2\right) V^{-}} \frac{a_{N+1}}{r} \frac{r_{q}}{a_{1}},
\end{aligned}
$$

where

$$
V^{+}=k a_{N+1} A^{+}+\left(1+2 k^{2} a_{N+1}^{2} P_{0}\right) B^{+} \quad V^{-}=k a_{N+1} A^{-}+\left(1+2 k^{2} a_{N+1}^{2} P_{0}\right) B^{-},
$$

with $P_{0}=\ln \left(k a_{N+1} / 2 \gamma\right)+C_{E}, C_{E}=0.577216$ is the Euler constant. In the ultrarelativistic $\operatorname{limit}(\ln \gamma \rightarrow \infty)$, the external longitudinal dipole electric and magnetic fields are vanishing. In the inner region of the pipe, we get $V^{+} / V^{-}=$ $B^{+} / B^{-}$. Note that, as the monopole $(m=0)$ and dipole $(m=1)$ terms of the external longitudinal electric and magnetic fields vanish in the ultrarelativistic limit, the Pointing vectors for these modes are parallel to the tube axis in the outer region.

The high multipole components $(m>1)$ of the longitudinal electric field in the ultrarelativistic approximation $(\gamma \rightarrow \infty)$ are derived as

$$
\begin{aligned}
& E_{z m}^{\text {in }}=-c B_{z m}^{\text {in }}=-j \frac{q Z_{0} k}{\pi}\left(\frac{k^{2} a_{1}^{2}}{m+1}-m+k a_{1} U^{+} / U^{-}\right)^{-1}\left(\frac{r_{q}}{a_{1}}\right)^{m}\left(\frac{r}{a_{1}}\right)^{m} \\
& E_{z m}^{\text {out }}=c B_{z m}^{\text {out }}=j \frac{q Z_{0} k}{2 \pi} \frac{\left(m^{2}-1\right) k a_{N+1}}{(m+1) k a_{1} U^{+}+\left[k^{2} a_{1}^{2}-m(m+1)\right] U^{-}}\left(\frac{a_{N+1}}{r}\right)^{m}\left(\frac{r_{q}}{a_{1}}\right)^{m},
\end{aligned}
$$

where

$$
\begin{aligned}
& U^{+}=(m-1) k a_{N+1} A^{+}+\left[m(m-1)-k^{2} a_{N+1}^{2}\right] B^{+} \\
& U^{-}=(m-1) k a_{N+1} A^{-}+\left[m(m-1)-k^{2} a_{N+1}^{2}\right] B^{-} .
\end{aligned}
$$

Coefficients $A^{ \pm}, B^{ \pm}$in (32) and (34) are defined via the elements of the field transformation matrix as

$$
\begin{aligned}
& A^{+}=\operatorname{det}\left[\hat{h}_{11 ; 22}+\hat{h}_{33 ; 44}+j\left(\hat{h}_{13 ; 24}-\hat{h}_{31 ; 42}\right)\right], \\
& B^{+}=\operatorname{det}\left[\hat{h}_{11 ; 23}-j \hat{h}_{31 ; 4}\right], \\
& A^{-}=\operatorname{det}\left[\hat{h}_{21 ; 42}+j \hat{h}_{23 ; 44}\right], \quad B^{-}=\operatorname{det}\left[\hat{h}_{21 ; 43}\right],
\end{aligned}
$$

where the matrix $\hat{h}_{p i ; q j}$ is composed as

$$
\hat{h}_{p i ; q j}=\left\{\begin{array}{ll}
Q_{p i} & Q_{p j} \\
Q_{q i} & Q_{q j}
\end{array}\right\}
$$

\section{IMPEDANCES}

The longitudinal $Z_{\|}(\omega, r, \theta)$ and transverse $\vec{Z}_{\perp}(\omega, r, \theta)$ impedances are defined as a Fourier transformation of the normalized longitudinal and transverse components of the integrated Lorenz force (wake potentials) and contain only the synchronous components of the fields [2]:

$$
\begin{aligned}
Z_{\|}(\omega, r, \theta) & =-\frac{1}{q} E_{z}^{\mathrm{in}}(\omega, r, \theta), \\
\vec{Z}_{\perp}(\omega, r, \theta) & =-\frac{j}{q}\left(\vec{E}_{\perp}^{\mathrm{in}}(\omega, r, \theta)+\left[\vec{v} \times \vec{B}^{\mathrm{in}}(\omega, r, \theta)\right]_{\perp}\right) .
\end{aligned}
$$

The dimensions of the longitudinal and transverse impedances per unit length are $\mathrm{Ohm} / \mathrm{m}$. Thus the $m$-pole com- ponent of impedances are derived as

$$
\begin{aligned}
Z_{\|}^{(m)}(\omega, r, \theta) & =-\frac{1}{q}\left[\tau I_{m}(\lambda r) D_{2}-E_{z}^{(p)}(r)\right] \cos m \theta \\
Z_{r}^{(m)}(\omega, r, \theta) & =-\frac{j}{q}\left(E_{r m}^{\mathrm{in}}-v B_{\theta m}^{\mathrm{in}}\right) \\
& =-\frac{\tau^{2}}{q}\left[D_{2} I_{m}^{\prime}(\lambda r)-j E_{r}^{(p)}(r)\right] \cos (m \theta) \\
Z_{\theta}^{(m)}(\omega, r, \theta) & =-\frac{j}{q}\left(E_{\theta m}^{\mathrm{in}}+v B_{r m}^{\mathrm{in}}\right) \\
& =\frac{\tau}{q}\left[D_{2} m \frac{I_{m}(\lambda r)}{k r}-j \tau E_{\theta}(p)\right] \sin (m \theta)
\end{aligned}
$$

that satisfy the Panowsky-Wenzel theorem (see, for example [2]):

$$
\nabla_{\perp} Z_{\|}^{(m)}=k \vec{Z}_{\perp}^{(m)} .
$$

The corresponding ultrarelativistic approximation of the impedances is then expressed as

$$
\left.\begin{array}{rl}
Z_{\|}^{(m)} & =\tilde{Z}_{\|}^{(m)}(k)\left(\frac{r}{a_{1}}\right)^{m}\left(\frac{r_{q}}{a_{1}}\right)^{m} \cos m \theta \\
Z_{r}^{(m)} \\
Z_{\theta}^{(m)}
\end{array}\right\}=\tilde{Z}_{T}^{(m)}(k)\left(\frac{r}{a_{1}}\right)^{m-1}\left(\frac{r_{q}}{a_{1}}\right)^{m}\left\{\begin{array}{l}
\cos m \theta \\
-\sin m \theta,
\end{array}\right.
$$

where the frequency dependent part of impedances $\tilde{Z}_{\|}^{(m)}(k)$, $\tilde{Z}_{T}^{(m)}(k)$ is derived as 


$$
\tilde{Z}_{\|}^{(m)}(k)= \begin{cases}j \frac{Z_{0}}{\pi k a_{1}^{2}}\left(1-\frac{2}{k a_{1}} \frac{Q_{33}}{Q_{23}}\right)^{-1} & \text { for } m=0 \\ j \frac{Z_{0}}{\pi} k\left(\frac{k^{2} a_{1}^{2}}{m+1}-m+k a_{1} G_{m}\right)^{-1} & \text { for } m \geq 1\end{cases}
$$

$$
\tilde{Z}_{T}^{(m)}(k)=\frac{m}{k a_{1}} \tilde{Z}_{\|}^{(m)}(k)
$$

with $G_{1}=V^{+} / V^{-}$for the dipole mode $(m=1)$ and $G_{m}=$ $U^{+} / U^{-}$for high multipoles $(m>1)$.

For the two-layer tube the monopole term of impedance (41) coincides with the analytical expressions obtained in [20]. In the ultrarelativistic approximation of the dipole mode in (41), we kept the asymptotic presentation (31). In the ultrarelativistic limit $[\ln (\gamma) \rightarrow \infty]$ we get $V^{+}=U^{+}$, $V^{-}=U^{-}$for $m=1$ and the multipole impedance $(m \geq$ 1) of (41) for two-layer tube coincides with the analytical expressions obtained in [21].

For the three-layer tube the asymptotic analytical expression for the longitudinal monopole impedance can be derived for the frequency range when the skin depths of the layers are smaller than the inner radius of the tube:

$$
\begin{aligned}
\tilde{Z}_{\|}^{(0)}= & j \frac{Z_{0}}{\pi k a_{1}^{2}}\left(1+\frac{2}{k a_{1}} \frac{\varepsilon_{1} k}{\varepsilon_{0} \chi_{1}}\right. \\
& \left.\times \frac{\operatorname{th}\left(\chi_{1} d_{1}\right)+\Psi\left(d_{3}, d_{2}\right)}{1+\operatorname{th}\left(\chi_{1} d_{1}\right) \Psi\left(d_{3}, d_{2}\right)}\right)^{-1},
\end{aligned}
$$

where

$$
\Psi\left(d_{3}, d_{2}\right)=\frac{\varepsilon_{2} \chi_{1}}{\varepsilon_{1} \chi_{2}} \frac{\varepsilon_{2} \chi_{3} \operatorname{th}\left(\chi_{3} d_{3}\right) \operatorname{th}\left(\chi_{2} d_{2}\right)+\varepsilon_{3} \chi_{2}}{\varepsilon_{2} \chi_{3} \operatorname{th}\left(\chi_{3} d_{3}\right)+\varepsilon_{3} \chi_{2} \operatorname{th}\left(\chi_{2} d_{2}\right)} .
$$

In (43) and (44) $d_{1}, d_{2}, d_{3}$ are the layer thicknesses. At $d_{1}=0$ or $d_{3}=0$, expression (43) passes to the two-layer tube impedance [20]. For $d_{1} \rightarrow \infty\left[\operatorname{th}\left(\chi_{1} d_{1}\right) \rightarrow 1\right]$, the impedance is converted to the classical impedance of the single-layer tube with infinite wall thickness [1].

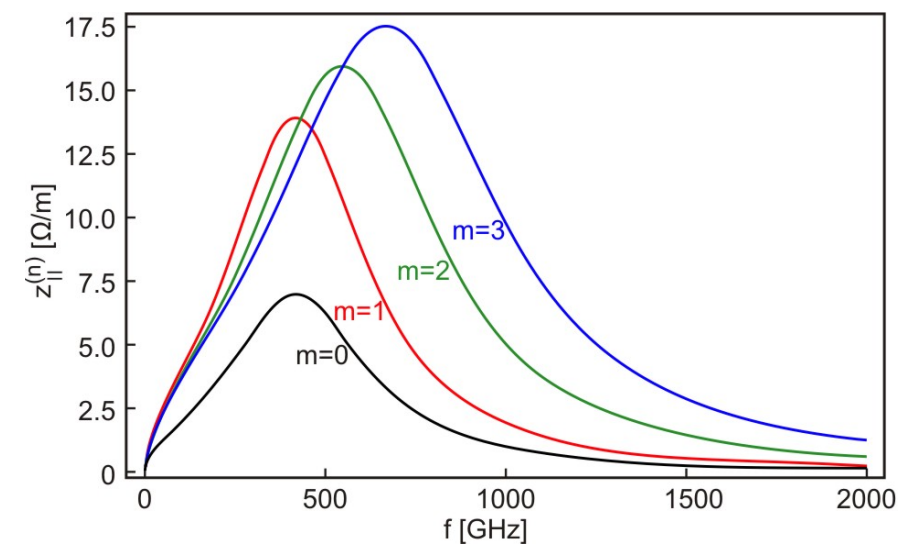

\section{NUMERICAL EXAMPLES FOR PETRA III VACUUM CHAMBER}

The special cases of the results given in previous sections are the impedances of finite-thickness single and twolayer tubes. In this section we present the numerical examples of the impedance calculation for the PETRA III storage ring straight section and wiggler vacuum chambers [4,34].

The standard straight section vacuum chamber is the stainless steel tube of circular cross section with $47 \mathrm{~mm}$ inner radius and wall thickness of $2 \mathrm{~mm}$. Figures 2 and 3 present the frequency dependent part of longitudinal $\tilde{Z}_{\|}^{(m)}(k)$ and transverse $\tilde{Z}_{T}^{(m)}(k)$ multipole impedances for the standard straight section vacuum chamber. For a wall thickness of $2 \mathrm{~mm}$, the skin depth is equal to the wall thickness when the frequency is equal to $f_{0} \sim 42 \mathrm{KHz}$. For frequencies $f \gg f_{0}$, the results coincide with the infinite-thickness round tube impedance given by Chao [1].

The wiggler vacuum chamber is an aluminum chamber with internal NEG (nonevaporable getter) coating. The geometry of the chamber has an elliptical cross section with major and minor radii of $a=48 \mathrm{~mm}$ and $b=$ $8.95 \mathrm{~mm}$, respectively, the thickness of the chamber is $2.8 \mathrm{~mm}$.

The longitudinal and transverse impedances of the elliptical vacuum chamber are well approximated by the impedances of round chamber (with radius $b$ ) multiplied by the geometrical factors that depend on the aspect ratio $a / b$. For the large aspect ratio $a / b>2$, the geometrical factor of the longitudinal impedance is equal to unity, the horizontal geometrical factor vanishes and the vertical geometrical factor is given by $\pi^{2} / 8=1.23$ [35].

Figure 4 presents the real and imaginary parts of the vertical dipole impedance of the PETRA III wiggler vacuum chamber for the NEG thickness of 0.5 and $1 \mu \mathrm{m}$ in round chamber approximation. For the NEG thickness of $1 \mu \mathrm{m}$, the frequency that corresponds to the skin depth

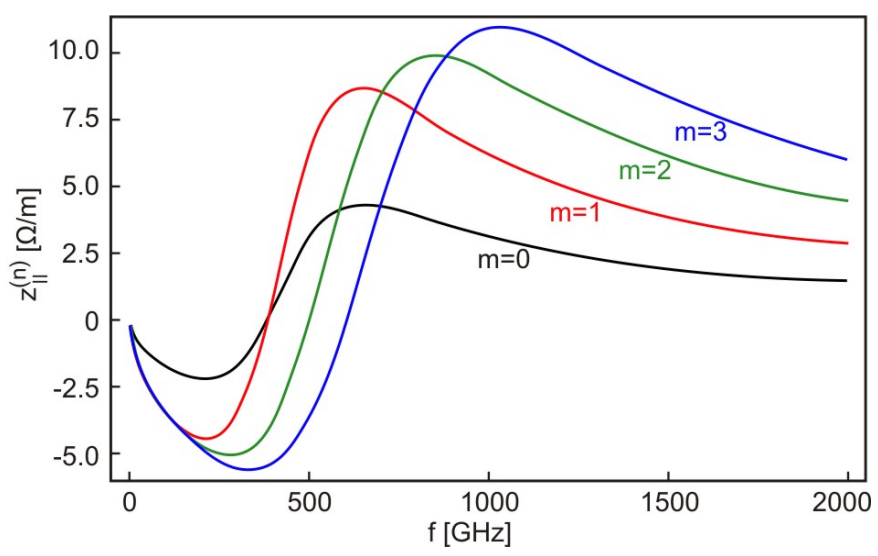

FIG. 2. (Color) Real (left) and imaginary (right) parts of longitudinal multipole impedances for PETRA III vacuum chamber. Standard straight section. 

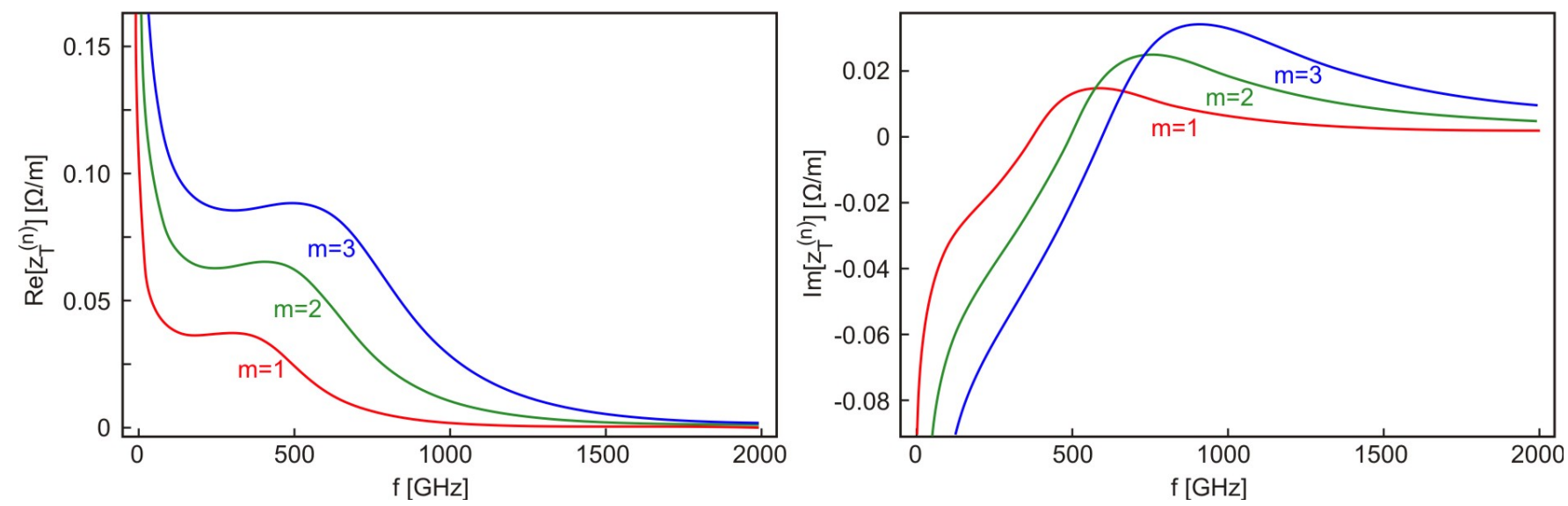

FIG. 3. (Color) Real (left) and imaginary (right) parts of transverse impedances for PETRA III vacuum chamber. Standard straight section.
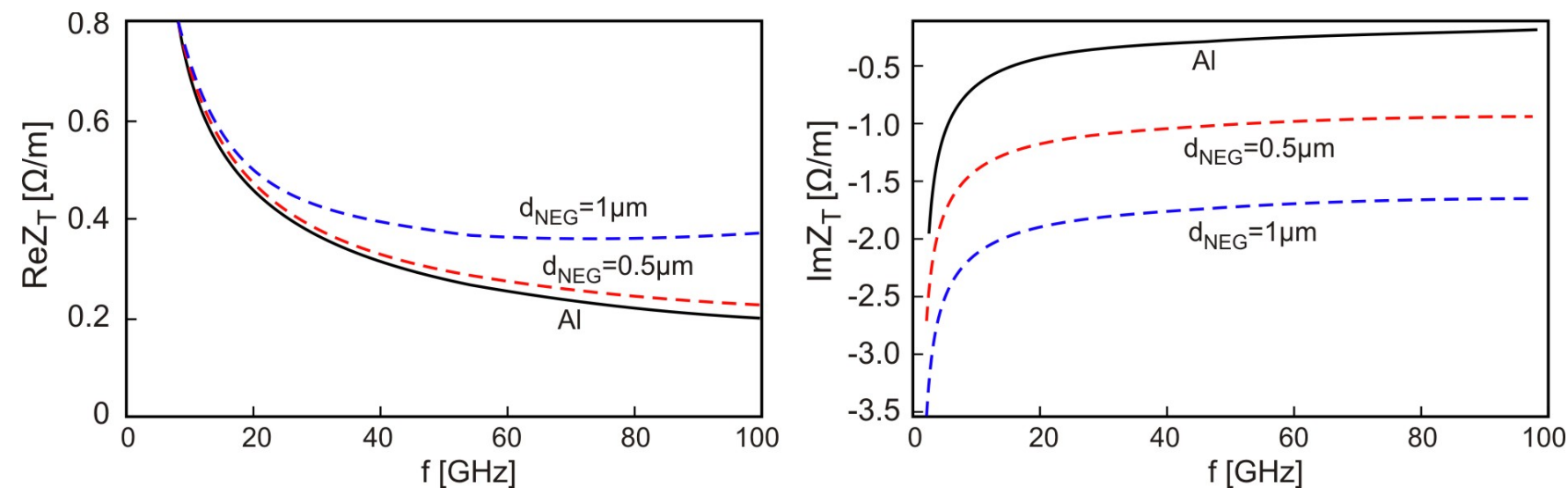

FIG. 4. (Color) Real (left) and imaginary (right) parts of the transverse dipole impedance for PETRA III wiggler vacuum chamber. Aluminum chamber with NEG coating.

equal to cover thickness is in order of $f_{0} \sim 820 \mathrm{GHz}$. For this case, as it can be seen from Fig. 4, the impact of the NEG coating is visible for frequencies $f \geq 5-10 \mathrm{GHz}$.

\section{NUMERICAL EXAMPLE FOR THREE- LAYER TUBE}

In this section we present the numerical example for longitudinal monopole impedance of the three-layer tube in the ultrarelativistic limit. Tube inner and outer radii are taken 5 and $6 \mathrm{~mm}$, respectively. The layer materials are (from external to inner layer): stainless steel (SS), copper $(\mathrm{Cu})$, and nonevaporable getter (NEG) with corresponding static conductivities $\sigma_{\mathrm{SS}}=0.14 \times 10^{7} \Omega^{-1} \mathrm{~m}^{-1}, \sigma_{\mathrm{Cu}}=$ $5.88 \times 10^{7} \Omega^{-1} \mathrm{~m}^{-1}$, and $\sigma_{\mathrm{NEG}}=0.55 \times 10^{5} \Omega^{-1} \mathrm{~m}^{-1}$. Figure 5 presents the three-layer tube longitudinal impedances for various thicknesses of the NEG layer $(0.01-10 \mu \mathrm{m})$ with intermediate copper coating of $1 \mu \mathrm{m}$ thickness. The thick smooth curves in the figure present the impedances of stainless steel, copper, and NEG singlelayer tubes with infinite wall thickness and tube radii of
6,5 , and $5 \mathrm{~mm}$, respectively. As a longitudinal coordinate the dimensionless wave number $k_{0}=k s_{0}$ is used with characteristic parameter $s_{0}$ given as $s_{0}=$ $\left(2 \mathrm{ca}_{1}^{2} \varepsilon_{0} / \sigma_{\mathrm{Cu}}\right)^{1 / 3}[23]$.

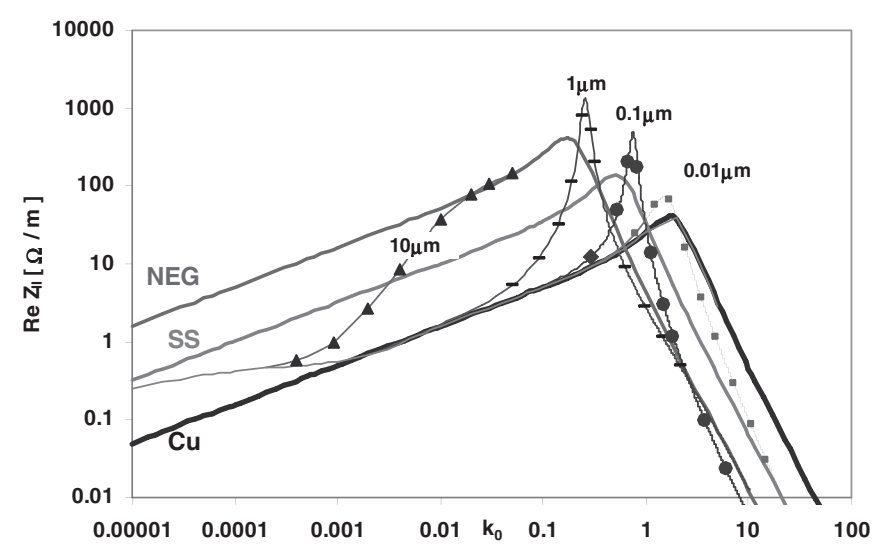

FIG. 5. Three-layer tube longitudinal monopole impedance for various NEG-layer thickness. Copper layer thickness is $1 \mu \mathrm{m}$. 
At very low frequencies, the three-layer tube impedance is conditioned by the outer SS-layer impedance due to field penetration, while the very high frequency part of the impedance is conditioned by the inner NEG-layer impedance. A characteristic high frequency peak is observed for inner layer thickness of about $1 \mu \mathrm{m}$. The similar peak is observed for a two-layer tube [20]. Note that the impedance peak is correlated with the inner layer thickness.

\section{CONCLUSION}

In this paper we described the method of calculating the longitudinal and transverse impedances of the laminated round pipe with many layers of different materials. The matrix formalism has been developed to describe the field transitions through the subsequent layers that allow coupling the electromagnetic fields inside and outside the pipe. The number of equations to be solved is then reduced to four algebraic equations. The solutions and ultrarelativistic approximations for the field harmonics in the inner and outer regions of the pipe are derived. The multipole longitudinal and transverse impedances are evaluated. The numerical examples for the single-, two-, and three-layer vacuum chambers are presented.

\section{ACKNOWLEDGMENTS}

The authors express thanks to Ursula van-Rienen, Dirk Hecht, Klaus Balewski, Winfried Decking, and Rainer Wanzenberg for many stimulating discussions.

\section{APPENDIX A}

The expressions for coefficients $D_{k}(k=1,2,3,4)$ can be presented in convenient form as

$$
D_{i}=-j \frac{q}{\pi} \frac{Z_{0} h_{m} \beta}{a_{1}} \frac{I_{m}\left(\lambda r_{q}\right) G_{i}}{I_{m}\left(\lambda a_{1}\right) G}, \quad i=1,2,3,4,
$$

where

$$
\begin{aligned}
G_{1} & =\frac{j m}{\varsigma_{1}} F_{42}-\tau F_{41}, \\
G_{2} & =\frac{I_{m}^{\prime}\left(\mathrm{s}_{1}\right)}{I_{m}\left(\mathrm{~s}_{1}\right)} F_{42}+\tau F_{21}, \\
G_{3} & =-\tau\left(\tau I_{m}\left(\mathrm{~s}_{1}\right) \Pi_{1}-j m \frac{I_{m}\left(\mathrm{~s}_{1}\right)}{\varsigma_{1}} \Pi_{2}-I_{m}^{\prime}\left(\mathrm{s}_{1}\right) \Pi_{4}\right) \\
G_{4} & =-\tau\left(\tau I_{m}\left(\mathrm{~s}_{1}\right) \Pi_{1}^{\prime}-j m \frac{I_{m}\left(\mathrm{~s}_{1}\right)}{\varsigma_{1}} \Pi_{2}^{\prime}-I_{m}^{\prime}\left(\mathrm{s}_{1}\right) \Pi_{4}^{\prime}\right) \\
G & =\frac{j m I_{m}\left(\mathrm{~s}_{1}\right)}{\varsigma_{1}} G_{1}+\beta^{2} I_{m}^{\prime}\left(\mathrm{s}_{1}\right) G_{2}+\left(\Pi_{3}^{\prime} G_{3}-\Pi_{3} G_{4}\right),
\end{aligned}
$$

and
$\Pi_{k}=\left(j m \mathrm{~s}_{2}^{-1} Q_{k, 1}+\tau Q_{k, 2}\right) K_{m}\left(\mathrm{~s}_{2}\right)+\beta^{2} Q_{k, 3} K_{m}^{\prime}\left(\mathrm{s}_{2}\right)$

$\Pi_{k}^{\prime}=\left(-j m \mathrm{~s}_{2}^{-1} Q_{k, 3}+\tau Q_{k, 4}\right) K_{m}\left(\mathrm{~s}_{2}\right)+Q_{k, 1} K_{m}^{\prime}\left(\mathrm{s}_{2}\right)$

$F_{i j}=-\Pi_{i} \Pi_{j}^{\prime}+\Pi_{j} \Pi_{i}^{\prime}$

with $\mathrm{s}_{1}=\lambda a_{1}, \mathrm{~s}_{2}=\lambda a_{N+1}$, and $Q_{i j}$ the elements of field transformation matrix $\hat{Q}$.

[1] A. W. Chao, Physics of Collective Beam Instabilities in High Energy Accelerators (Wiley, New York, 1993).

[2] B. W. Zotter and S. A. Kheifetz, Impedances and Wakes in High-Energy Particle Accelerators (World Scientific, Singapore, 1997).

[3] Report No. DESY 2006-097, edited by M. Altarelli et al., 2006.

[4] PETRA III, Technical Design Report, Report No. DESY 2004-035, edited by K. Balewski, W. Brefeld, W. Decking, H. Franz, R. Rohlsberger, and E. Weckert, 2004.

[5] V. Tsakanov et al., Rev. Sci. Instrum. 73, 1411 (2002).

[6] J.M. Filhol et al., in Proceedings of the 10th European Particle Accelerator Conference, Edinburgh, Scotland, 2006 (EPS-AG, Edinburgh, Scotland, 2006), pp. 27232727.

[7] J. Wei, in Proceedings of the Workshop on Space Charge Physics in High Intensity Hadron Rings, Shelter Island, New York, 1998, AIP Conf. Proc. No. 448 (AIP, New York, 1998), p. 152.

[8] Y. Yamazaki et al., KEK Report No. 2002-13, 2003.

[9] M. Benedikt et al., in Proceedings of the European Particle Accelerator Conference, Vienna, 2000 (EPS, Geneva, 2000), pp. 527-529.

[10] D. Briggs, Report No. SSCL-512-REV1, 1992.

[11] F. Ruggiero, Part. Accel. 50, 83 (1995).

[12] R. Nagaoka, in Proceedings of the European Particle Accelerator Conference, Lucerne, 2004 (EPS-AG, Lucerne, 2004), pp. 2038-2040.

[13] I. Hoffmann, G. Franchetti, and A. Fedotov, AIP Conf. Proc. 642, 248 (2002).

[14] A. S. Vardanyan and G. G. Oksuzyan, Tech. Phys. 47, 448 (2002).

[15] A. Piwinski, IEEE Trans. Nucl. Sci. 24, 1364 (1977).

[16] R. Glukshtern and B. Zotter, Phys. Rev. ST Accel. Beams 4, 024402 (2001).

[17] X.E. Lin, Report No. SLAC-PUB-7924, 1998.

[18] A. M. Al-khateeb, O. Boine-Frankenheim, R. W. Hasse, and I. Hofmann, Phys. Rev. E 71, 026501 (2005).

[19] A. M. Al-khateeb, R. W. Hasse, O. Boine-Frankenheim, W. M. Daga, and I. Hofmann, Phys. Rev. ST Accel. Beams 10, 064401 (2007).

[20] M. Ivanyan and V. Tsakanov, Phys. Rev. ST Accel. Beams 7, 114402 (2004).

[21] M. Ivanyan and V. Tsakanian, Phys. Rev. ST Accel. Beams 9, 034404 (2006).

[22] N. Wang and Q. Qin, Phys. Rev. ST Accel. Beams 10, 111003 (2007).

[23] K. L. F. Bane and M. Sands, Report No. SLAC-PUB-957074, 1995.

[24] B. Zotter, Part. Accel. 1, 311 (1970). 
[25] B. Zotter, CERN Technical Report No. 69-15, 1969.

[26] A. Burov and V. Lebedev, Report No. FERMILAB-Conf02/100-T, 2002.

[27] J. D Jackson, Report No. SSC-N-110, 1986.

[28] E. Keil and B. Zotter, in Proceedings of the European Particle Accelerator Conference, Stockholm, 1998 (IOP, London, 1998), pp. 963-965.

[29] G. R. Lambertson, Reports No. LBL-44454 and No. LBNL-44454, 1999.

[30] A. Burov and A. Novokhatskii, Report No. 90-28, INPNovosibirsk, 1990.
[31] T. Perron, L. Farvacque, and E.Plouviez, in Proceedings of the European Particle Accelerator Conference, Lucerne, 2004 (EPS-AG, Lucerne, 2004), pp. 2053-2055.

[32] T.F. Gunzel, Phys. Rev. ST Accel. Beams 9, 114402 (2006).

[33] T. Weiland and R. Wanzwnberg, Report No. DESY M 9106, 1991.

[34] M. Ivanyan, E. Laziev, V. Tsakanov, A. Vardanyan, A. Tsakanian, and R. Wanzenberg, Report No. DESY M 0701, 2007, p. 26.

[35] A. Piwinski, Report No. DESY-94-068, 1994, p. 23. 